\title{
Study Site Planned Duration
}

National Cancer Institute

\section{Source}

National Cancer Institute. Study Site Planned Duration. NCI Thesaurus. Code C94150.

The intended period of time for the study site's participation in the study. 